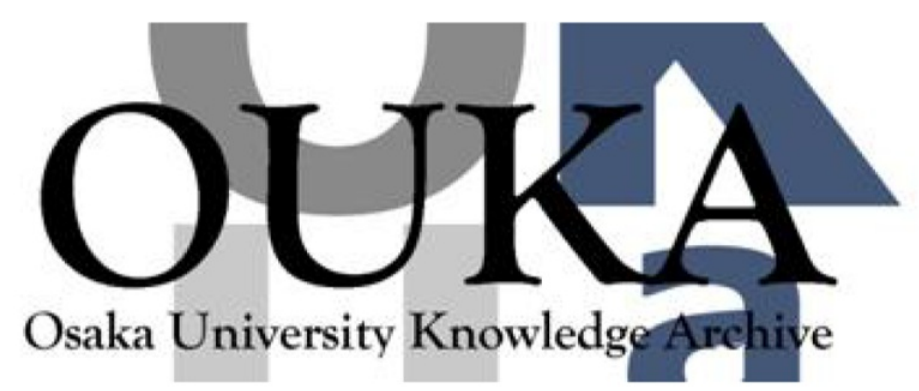

\begin{tabular}{|c|l|}
\hline Title & 10-kJ PW Laser for the FIREX-I Program \\
\hline Author(s) & Miyanaga, N.; Azechi, H. ; Tanaka, K. A. et al. \\
\hline Citation & Journal De Physique. IV : JP. 133 p. $81-p .87$ \\
\hline Issue Date & $2006-06$ \\
\hline oaire:version VoR \\
\hline URL & https://hdl. handle. net/11094/3320 \\
\hline rights & \\
\hline Note & \\
\hline
\end{tabular}

Osaka University Knowledge Archive : OUKA

https://ir. Library. osaka-u. ac. jp/

Osaka University 


\title{
10-kJ PW laser for the FIREX-I program
}

\author{
N. Miyanaga ${ }^{1}$, H. Azechi ${ }^{1}$, K.A. Tanaka ${ }^{2}$, T. Kanabe ${ }^{3}$, T. Jitsuno ${ }^{1}$, \\ J. Kawanaka ${ }^{1}$, Y. Fujimoto ${ }^{1}$, R. Kodama ${ }^{2}$, H. Shiraga ${ }^{1}$, K. Knodo ${ }^{2}$,

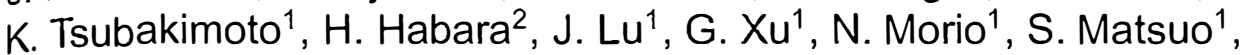 \\ E. Miyaji ${ }^{1}$, Y. Kawakami ${ }^{1}$, Y. Izawa ${ }^{1}$ and K. Mima ${ }^{1}$ \\ 1 Institute of Laser Engineering, Osaka University, Suita, Osaka 565-0871, Japan \\ 2 Graduate School of Engineering, Osaka University, Suita, Osaka 565-0871, Japan \\ ${ }^{3}$ Graduate School of Engineering, University of Fukui, Fukui, Fukui 910-8507, Japan
}

\begin{abstract}
A 10-kJ PW laser (LFEX) is under construction for the FIREX-I program. This paper reports a design overview of LFEX, the technological development of a large-aperture arrayed amplifier with modified four-pass architecture, wavefront correction, a large-aperture Faraday rotator with a superconducting magnet, a new pulse compressor arrangement, and focus control.
\end{abstract}

\section{INTRODUCTION}

Inlaser fusion research, the concept of fast ignition [1] by an ultra-intense laser holds great interest because high gain is feasible with relatively small driver energy. On the basis of experimental demonstrations of fast heating [2, 3] of deuterated plastic plasma by a PW laser [4], we have started phase I of the FIREX (Fast Ignition Realization Experiment) program, in which fuel imploded using the existing GEKKO XII will be heated by a high-energy PW laser. An important mission for FIREX-I is to achieve a heating temperature of $5-10 \mathrm{keV}$, which is a necessary condition for fuel ignition. Another important mission is the development of laser technology for phase II of FIREX, which will need an energy-upgrade of both the implosion and heating lasers to $50 \mathrm{~kJ}$ level.

The high-energy PW laser under construction for FIREX-I (LFEX: Laser for Fast Ignition Experiment) is a $\mathrm{Nd}$ : glass laser aiming for a $10-\mathrm{kJ}$ output energy. To achieve the $5-10 \mathrm{keV}$ temperature by overcoming the energy loss due to plasma expansion, the required pulse shape is a 10-ps trapezoid with a rise time less then 2 ps. The focal spot size should be smaller than $30 \mu \mathrm{m}$ with encircled energy larger than $50 \%$. In accord with these specifications, the system design has been undertaken in terms of a multi-pass amplifier for efficient energy extraction, operational conditions of chirped pulse amplification (CPA), which meets a realistic pulse compressor size, and a minimized wavefront distortion [5].

\section{DESIGN OVERVIEW OF LFEX}

Based on the analysis of the CPA characteristic bench-marked by data from the existing PW laser [4], the whole system has been designed as is schematically shown in Figure' 1. The LFEX laser system consists of four subsystems;

- the front-end subsystem, which is based on that of the existing PW laser,

- the preamplifier subsystem, which includes a four-pass rod amplifier, beam dividing optics and a two-stage rod amplifier,

- the main amplifier subsystem that uses a modified four-pass architecture, and

- the rear-end subsystem that consists of beam transport optics, pulse compressors and the final focusing optics. 


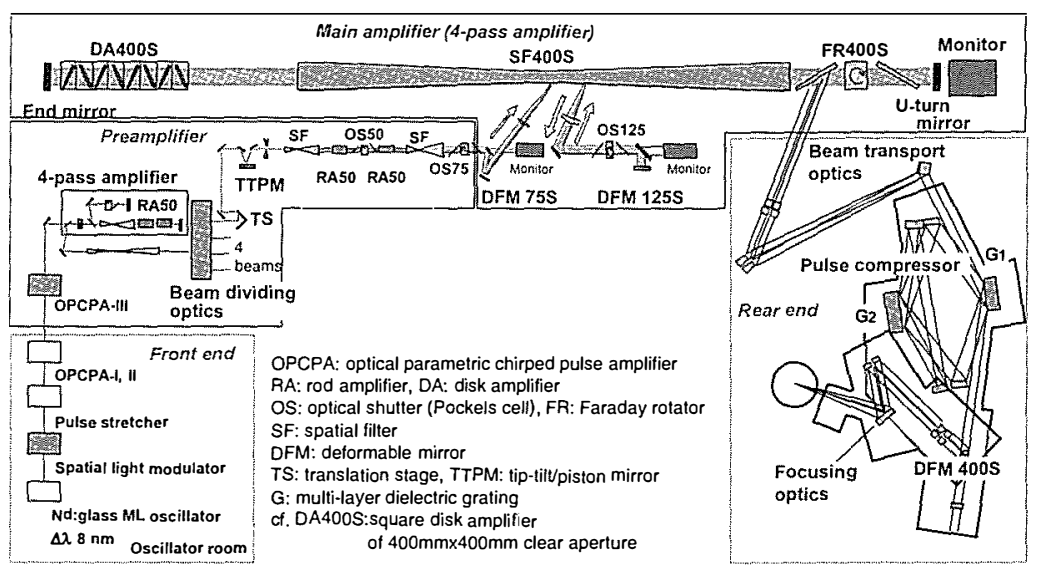

Figure 1. Block diagram of the LFEX laser system.

The required spectral width of the CPA output should be $\sim 3 \mathrm{~nm}$ to realize a 2-ps rise time after pulse compression. A constraint on the B integral within the amplifier requires a pulse width of $\sim 2 \mathrm{~ns}$ that corresponds to a chirping of $\sim 3 \mathrm{~nm} / 2 \mathrm{~ns}$. The main amplifier (disk amplifier: DA400S, where $400 \mathrm{~S}$ denotes a square clear aperture of $400 \mathrm{~mm} \times 400 \mathrm{~mm}$ ) uses NIF scale laser glass slabs [6], and the minimum number of main amplifier chains is 4 . Each four-pass main amplifier chain has a 50 -m long spatial filter (SF400S) that relays an image of a beam apodizer being set in the preamplifier through the pulse compressor. A large-aperture Faraday rotator (FR400S) is installed after the SF400S to protect the amplifier from back reflection from the target. The preamplifier and main amplifier subsystems have already been installed in the laser bay of GEKKO XII.

The rear-end subsystem has been designed to be set in the target room I of GEKKO XII. The specification for the grating-to-grating distance of the pulse compressor is less than $10 \mathrm{~m}$ for the doublepass arrangement. As is detailed later, the pulse compressor has been designed to use gratings from both sides to reduce cost and to compensate for angular deviation of the output beam caused by a groove density error of the tiled multi-layer dielectric (MLD) gratings.

\section{FRONT-END AND PREAMPLIFIER SUBSYSTEMS}

\subsection{Front end and optical chirped pulse amplifier}

The front end of the existing PW laser, which consists of a mode-locked Nd:glass laser (Time-Bandwidth Products, 150fs) and two stages of a pulse stretcher/optical parametric chirped pulse amplifier (OPCPA) [7], is modified to derive the chirping suitable for the compressor of the LFEX laser. To minimize the gain narrowing effect in the amplifier chain, an additional OPCPA stage has been introduced just in front of the preamplifier. This OPCPA derives $\sim 40-\mathrm{mJ}$ output energy with $\sim 6-\mathrm{nm}$ spectral width (chirping of $\sim 6 \mathrm{~nm} / 4 \mathrm{~ns}$ ) to ensure $\sim 3$-nm spectral width at an output energy of $\sim 3 \mathrm{~kJ} /$ chain after the main amplifier.

\subsection{Pulse shaping capability}

The spectral width of $\sim 3 \mathrm{~nm}$ was determined in terms of not only a practical size for the pulse compressor, but also the required rise time of the compressed pulse. Because the spectral shape of the $\sim 3-\mathrm{kJ}$ output is almost Gaussian, the minimum width of the transform-limited pulse is $\sim 0.5 \mathrm{ps}$. Therefore a 10 -ps trapezoid pulse with <2-ps rise time can be obtained by controlling the group velocity dispersion (GVD). Here we are reminded that the Gaussian-to-flat top beam homogenizer is an analogy in the spatial domain. 
The overall pulse elongation tol0ps can be easily done using the GVD mismatch between the stretcher and compressor (an artificial mismatch of grating-to-grating distances). Then, if we introduce a small, but appropriate GVD into a 10-ps compressed pulse, a nearly flat-top pulse can be obtained. For this a spectral phase modulator consisting of a diffraction grating and a spatial light modulator based on liquid crystal (PAL-SLM) has been installed in the front end. In addition, a small grating pair will be set in each beam line just after the beam dividing optics to introduce a desired stretcher-compressor mismatch and to compensate the imbalance of compressor path lengths ensuring the same pulse shape among the four beams.

\section{MAINAMPLIFIER SUBSYSTEM}

\subsection{Architecture of the main amplifier}

In high-energy $\mathrm{Nd}$ :glass lasers, far-field angle separation and polarization separation are utilized with two versions of multi-pass architecture, like a straight cavity (NIF [8]) and a L-turn cavity (LMJ [9] and SG-III [10]). In contrast to these laser systems, the FIREX-I laser uses longitudinal focus separation in conjunction with angle separation, as shown in Figure 2. A square beam of $68 \mathrm{~mm} \times 68 \mathrm{~mm}$ is injected at the back focal plane of the first path with a position shift of $\sim 1.5 \mathrm{~m}$ prior to the nominal far-field position of a spatial filter. This focal shift corresponds to a 0.5 -mrad beam divergence, which causes a reverse focal shift onto the fore focal plane of the returned (second) beam path. Using this angular-longitudinal focus separation, the beam size on a pick-up mirror can be enlarged without intersecting other beam paths; thus, a precision mirror coating with an extremely high damage threshold is not needed. The third and fourth paths are arranged in the same manner.

For polarization separation it is enough for a Pockels cell to act as a suppressor of parasitic oscillation, because a Faraday rotator is installed behind the output lens of the spatial filter (SF400S). Therefore, a Pockels cell with a clear aperture of $125 \mathrm{~mm} \times 125 \mathrm{~mm}$ is set just in front of the L-turn mirror (DFM125).

The main amplifier head consists of 8 units of $2 \times 2$ arrays. Each unit is designed as a motorized stack on a translational stage for maintenance. $2 \times 2$ arrays of NIF scale laser slabs in the unit are pumped by 27 flash lamps (43-mm bore diameter and $900-\mathrm{mm}$ discharge length) that are assembled as vertical arrays in two side and one central cassettes. The total stored energy of the capacitor bank is $\sim 6.5 \mathrm{MJ}$, and the overall laser efficiency is expected to be $\sim 0.4 \%$ for narrow band operation and $\sim 0.2 \%$ for CPA, respectively. Initial activation of the amplifier system (Figure 3 ) has been instituted, delivering an energy of $1.2 \mathrm{~kJ} / \mathrm{beam}$.

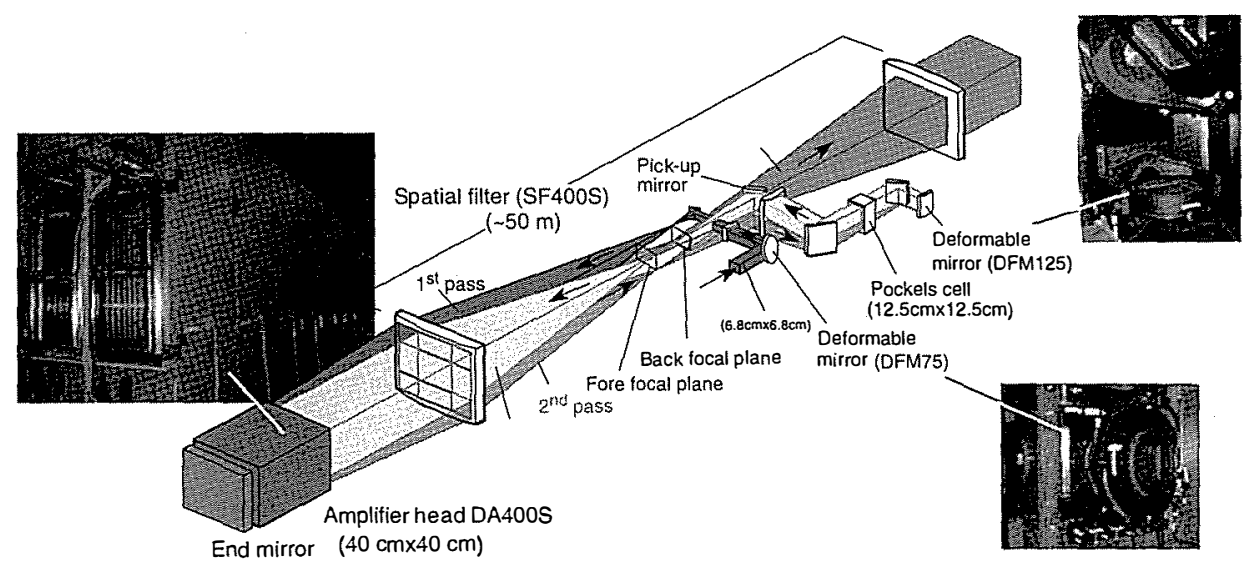

Figure 2. Schematic for a four-pass amplifier using angular-longitudinal focus separation. Only one beam is shown in the illustration. 


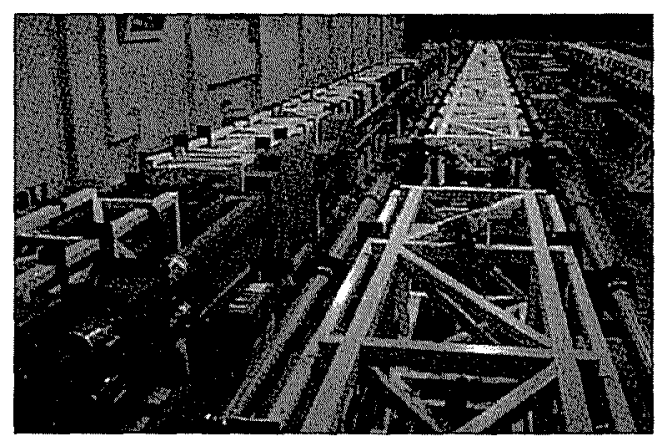

Figure 3. Amplifier chains of LFEX (left most lines).

\subsection{Compensation of wavef ront aberration}

Beam diagnostics and focusing control are important for a multi-beam PW laser system. In the main amplifier, the wavefront distortion caused in the first and second passes is corrected by a deformable mirror (DFM75 set at a conjugate plane of an end mirror of the four-pass main amplifier) that is controlled according to data from a Shack-Hartman sensor. This S-H sensor is being set at another conjugate plane near the DFM125. In the same manner, the DFM125 acts as a compensator for the third and fourth passes with the help of another S-H sensor being set behind the Faraday rotator.

\subsection{Large-aperture Faraday rotator}

The technology for protecting the amplifier from back reflection from a target is a crucial issue for a highenergy laser system. For this, a Faraday rotator that uses a superconducting magnet is the most reliable device in terms of fail-safe protection. Further, a large-bore, cryogen-free superconducting magnet, which is mature technology in the field of medical magnetic resonance imaging, is cost effective compared with the more usual pulsed magnet driven by a large capacitor bank. Figure 4 shows a photograph of a Faraday rotator of $1.25-\mathrm{m}$ clear aperture that covers the $2 \times 2$ beam arrays of the FIREX-I laser. The measured nonuniformity of the magnetic field is $\sim 10 \%$ (peak to valley) within a volume of $125 \mathrm{~cm} \times 8 \mathrm{~cm}$ where the $2 \times 2$ arrays of Faraday glasses are positioned. The $2 \times 2$ arrays of polarizers with quartz substrate are set in front of and behind these Faraday glasses. The beams passing through the Faraday rotator are reflected back by $2 \times 2$ mirror arrays, and the beams with vertical polarization are took out by front polarizers from the amplifier chain to the rear-end subsystem.

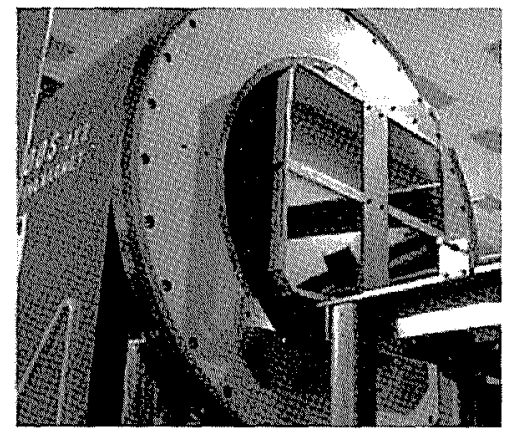

Figure 4. FR400S and arrayed Faraday glasses. 


\section{REAR-END SUBSYSTEM}

\subsection{Optimized arrangement of two tiled gratings for pulse compressor}

The grating arrangement of the pulse compressor (diamond-shaped compressor) has been fixed as shown schematically in Figure 5(a). The beam hits in turn $G_{1}$ (the first grating), $M_{3}$ (mirror), $G_{2}$ (the second grating), $M_{4}, M_{5}, G_{2}, M_{6}$, and $G_{1}$. If we use many tiles for each grating, this arrangement seems to be very sensitive to the groove density reproducibility. The diffraction angle deviation caused by groove density error is $\sim 4.6 \mu \mathrm{rad} / \mathrm{ppm}$ for a groove density of $1 / 1740 \mathrm{~mm}^{-1}$. Therefore, groove density error should be less than $0.1 \mathrm{ppm}$ for our focusing parabola of $4-\mathrm{m}$ focal distance. This groove density reproducibility may be realized using temperature-controlled fused silica as a grating substrate.

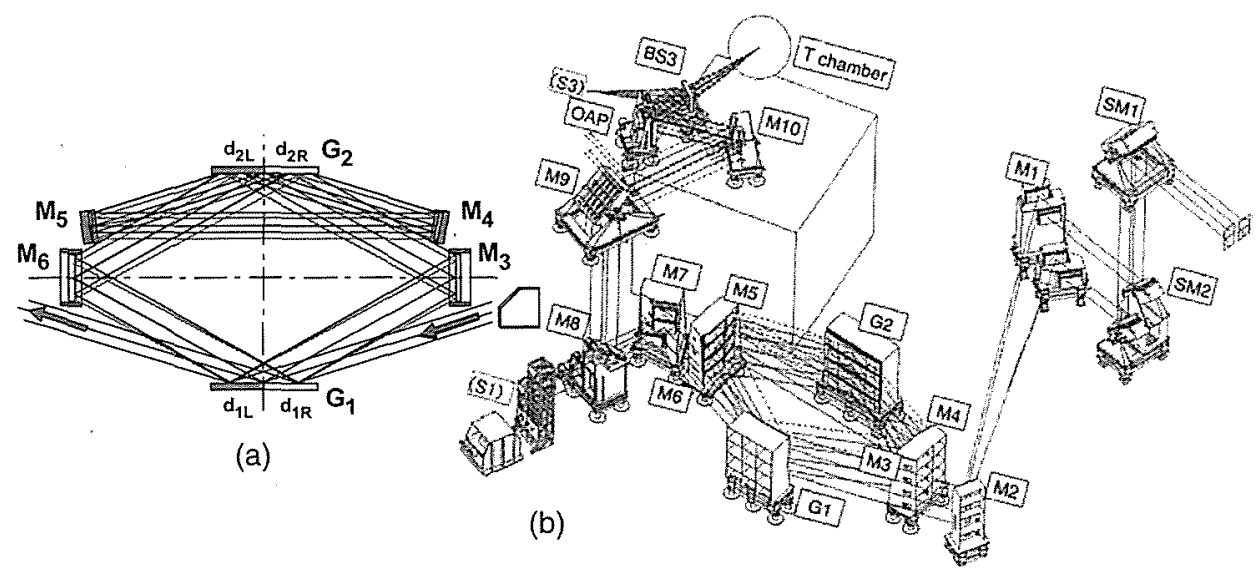

Figure 5. (a): Compressor arrangement using two tiled gratings. (b): Mechanical layout of the rear-end subsystem. G: grating, M: mirror, SM: stair mirror, OAP: off-axis parabola mirror, S: sensor, d: groove period of grating.

ILE-LLNL collaborative analysis of the grating arrangement showed that this diamond arrangement delivers a great advantage if we use gratings of two-tiled composite [11, 12]. In Figure 5(a) we have four cases of beam paths, i.e., $G_{1 R}-G_{2 L}-G_{2 R}-G_{1 L}, G_{1 R}-G_{2 R}-G_{2 L}-G_{1 L}, G_{1 L}-G_{2 L}-G_{2 R}-G_{1 R}$ and $G_{1 L}-G_{2 R}-G_{2 L}-$ $G_{I R}$, where subscript $1 R$, for example, denotes the right tile of the first grating. From a simple analysis assuming the complete parallelism of $G_{1}$ and $G_{2}$, we get a following formula for an angular deviation that depends on the groove period error $\delta_{\mathrm{IR}}, \delta_{1 \mathrm{~L}}, \delta_{2 \mathrm{R}}$ and $\delta_{2 \mathrm{~L}}$, where $d_{\mathrm{IR}}$, for example, in Figure 5(a) is $d_{1 \mathrm{R}}=d_{0}+\delta_{1 \mathrm{R}}$,

$$
\begin{aligned}
\Delta \theta\left(\delta_{\mathrm{IR}}, \delta_{\mathrm{IL}}, \delta_{2 \mathrm{R}}, \delta_{2 \mathrm{~L}}, \lambda\right) & =\frac{\lambda}{\cos \theta_{\mathrm{i} 0}}\left[\frac{1}{d_{0}+\delta_{\mathrm{IR}}}+\frac{1}{d_{0}+\delta_{1 \mathrm{~L}}}-\left(\frac{1}{d_{0}+\delta_{2 \mathrm{R}}}+\frac{1}{d_{0}+\delta_{2 \mathrm{~L}}}\right)\right] \\
& \approx \frac{-\lambda}{d^{2} \cos \theta_{\mathrm{i} 0}}\left[\left(\delta_{\mathrm{IR}}+\delta_{\mathrm{IL}}-\left(\delta_{2 \mathrm{R}}+\delta_{2 \mathrm{~L}}\right)\right]\right.
\end{aligned}
$$

for all of four cases of beam paths, where $\lambda$ and $\theta_{i 0}$ are wavelength and incident angle to the first grating, respectively. This advantage originates from the fact that the angular deviations caused by groove density error does not accumulate because the sign of angular deviation (diffraction angle deviation of $\mathrm{G}_{1}$ and $\mathrm{G}_{2}$ ) is reversed in the second incident onto $\mathrm{G}_{2}$ (incident angle deviation) by introducing two mirrors $\mathrm{M}_{4}$ and $\mathrm{M}_{5}$.Then, the angular shift is the same in the whole beam. Fortunately, the influence of the spectral band width is as small as $\Delta \theta \times 10^{-3} / \mathrm{nm}$. In addition, the phase jump caused by the groove density error can be eliminated by the parallel shift of the grating tiles, and finally the beam path of a compressed pulse is distinguished from the 0 -th order reflection from $G_{1}$ by slightly tilting $M_{5}$. 
The required size of grating is $420 \mathrm{~mm} \times 1800 \mathrm{~mm}$ (actually two pieces of tiled grating) for $\mathrm{G}_{2}$ which is determined by the chirped pulse width and the spectral width to be covered $(\sim \pm 1.5 \mathrm{~nm} \times 3=\sim \pm 4.5 \mathrm{~nm})$. The supply of such a large-aperture MLD grating with a high damage threshold has been realized based on recent rapid developments in the field of optical manufacturing technology.

\subsection{Preliminary test results of the tiled gratings}

The test of the two-tiled gratings was done using a Ti:sapphire laser in terms of dependences of far-field pattern and pulse shape on the phase jump between two tiles [5].

We are now concentrating on the development of stable and precision mechanics for assembling $420 \mathrm{~mm} \times 910 \mathrm{~mm}$ grating tiles. Figure 6 shows a photograph of sub-scale mechanics [13] for $200 \mathrm{~mm} \times 400 \mathrm{~mm}$ grating tiles. The test results of the driving characteristics for actuator and mechanical stability will be fed-back to the LFEX compressor.

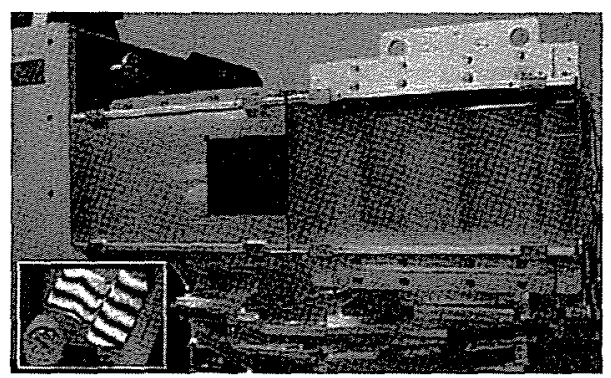

Figure 6. Prototype mechanics of tiled grating assembly holding a $200 \mathrm{~mm} \times 400 \mathrm{~mm}$ grating and a small reference grating.

\subsection{Compressor chamber and focusing optics}

A compressor chamber that covers many optics (from $G_{1}$ through the final focusing parabola in Figure 5(b)) will be installed by the end of March, 2006. The structure of the pulse compressor is a $\mathrm{x} \times 4$ vertical array. Therefore $2 \times 2$ beam arrays delivered from the Faraday rotator are transformed to a $1 \times 4$ array, and the $1 \times 4$ array of the output of the pulse compressor is again transformed to $2 \times 2$ arrays before reaching the monolithic focusing parabola.

Although several options are possible for focusing the four beams (a temporal stacking of 2-3 ps pulses on a target, for example), coherent addition of four beams is one of the great challenges in the FIREX PW laser. This requires short range (in the order of $100 \mathrm{~ms}$ ) phase locking of four beams. Because the elimination of static beam-to-beam phase jumps is relatively easy, a major residual source of phase variation is the rapid vibration of the catoptric elements. We are designing a real-time diagnostics method for beam pointing and phase variation, because mirror vibrations cause not only pointing errors but also significant changes in the path length. A tip/tilt-piston mirror will be implemented in upper stream of each main amplifier chain for the feed-back and feed-forward controls.

The phase aberration within the rear-end subsystem will also be corrected by deformable mirrors (M9 in Figure 5(b)). Although phase locking and deformable mirrors are a future option, the detailed design for a coherent combination of high-quality beams will be revised according to the measured characteristics of the four beams after construction of the main body of the rear-end subsystem. 


\section{SUMMARY}

The LFEX laser system for phase I of the FIREX program is under construction. Extensive studies have already been carried for the design of a 10-kJ PW laser. The installation of subsystems from the front end through the main amplifier chains, and some of the parts of the rear-end subsystem have been completed. First light from the main amplifier has been achieved, generating $1.2 \mathrm{~kJ} / \mathrm{beam}$. Now, an experimental campaign for CPA characterization is being carried out. In respect to the rear-end subsystem, the delivery of mount mechanics, mirrors and gratings will begin early 2006, and will finish in 2007. The first experimental campaign of plasma heating is expected to start in the middle of 2007 using part of the four beams.

\section{Acknowledgments}

The authors would like to gratefully acknowledge LLNL (especially Dr. J. H. Campbell and T. Baisden), DOE and MEXT Japan for their kind efforts in respect to the laser glass. The authors also deeply thank Mr. M.Rushford and Dr. C. Barty for fruitful discussions on arrayed gratings. Finally, the authors wish to acknowledge the great contribution of the many companies (especially LFT and Melco) and staff members involved in this project.

\section{References}

[1] M. Tabak, et al., Phys. Plasmas, 1 (1994) 1626-1634.

[2] R. Kodama, et al., Nature, 412 (2001) 798-802.

[3] R. Kodama, et al., Nature, 418 (2002) 933-934.

[4] Y. Kitagawa, et al.; IEEE J. Quant. Electron., 40 (2004) 281-293.

[5] N. Miyanaga, et al., Inertial Fusion Sciences and Applications 2003 (AṄS, 2004) pp. 507-511.

[6] J. Campbell, et al., J. Non-Crystal. Solids, 263\&264 (2000) 342-357.

[7] H. Yoshida, et al., Opt. Lett., 28 (2003) 257-259.

[8] E. Moses, Inertial Fusion Sciences and Applications 2003 (ANS, 2004) pp. 535-540.

[9] C. Cavailler, et al., Inertial Fusion Sciences and Applications 2003 (ANS, 2004) pp. 523-528.

[10] F. Jing, et al., "Preliminary Experiment Results of Technical Integration Line (TIL) for SG-III Laser Facility", China-Japan Seminar on Laser Fusion Science and High Energy Density Plasma Physics, Sanya Hainan, China, December 1-4, 2004; W. Zheng, Proceedings of this conference (FO25.1).

[11] T. Jitsuno, et al., Proceedings of this conference (PD-01).

[12] M. C. Rushford, et al., to be reported elsewhere.

[13] G. Xu, R. Kodama, et al, to be reported elsewhere. 